\title{
Revisiting Hypertext Infrastructure
}

\author{
Claus Atzenbeck ${ }^{*}$ \\ Hof University \\ Institute of Information Systems \\ Alfons-Goppel-Platz 1 \\ 95028, Hof, Germany \\ claus.atzenbeck@iisys.de
}

\author{
Thomas Schedel \\ Hof University \\ Institute of Information Systems \\ Alfons-Goppel-Platz 1 \\ 95028, Hof, Germany \\ thomas.schedel@iisys.de
}

\author{
Manolis Tzagarakis \\ University of Patras \\ Department of Economics \\ 26504, Rion, Greece \\ tzagara@upatras.gr
}

\author{
Daniel Roßner \\ Hof University \\ Institute of Information Systems \\ Alfons-Goppel-Platz 1 \\ 95028, Hof, Germany \\ daniel.rossner@iisys.de
}

\author{
Lucas Mages \\ Hof University \\ Institute of Information Systems \\ Alfons-Goppel-Platz 1 \\ 95028, Hof, Germany \\ lucas.mages@hof-university.de
}

\begin{abstract}
Specialized systems aiming at offering hypertext functionality in users' computing have been discussed since the early days of hypertext. However, with the claim to also support other structure domains than node-link structures, hypertext systems had to overcome some challenges. Researchers came up with component-based approaches and low level structure services.

Due to the raising omnipresence of the Web, research on traditional hypertext systems has been fading out over the past decade. This paper focuses again on hypertext infrastructures and goes beyond ongoing Web discussions. Based on lessons learned from well thought through previous work, we present a novel design for multi-structure supporting, general purpose hypertext systems that can be used in a series of application domains. The system provides intelligence analysis which is needed for sophisticated user support. We argue that this lets us use the hypertext system also as a visual analytics tool. Furthermore, for demonstration purposes we describe the use of the system in combination with a Web-based software engineering platform, which is part of the ongoing project ODIN.
\end{abstract}

\section{CCS CONCEPTS}

-Human-centered computing $\rightarrow$ Hypertext / hypermedia; -Software and its engineering $\rightarrow$ Software infrastructure;

\section{KEYWORDS}

hypertext infrastructure; open hypermedia systems; CB-OHS; spatial hypertext; navigational hypertext; taxonomic hypertext; Asgard

\footnotetext{
${ }^{*}$ Corresponding author
}

Permission to make digital or hard copies of part or all of this work for personal or classroom use is granted without fee provided that copies are not made or distributed for profit or commercial advantage and that copies bear this notice and the full citation on the first page. Copyrights for third-party components of this work must be honored. For all other uses, contact the owner/author(s).

HT'17, July 4-7, 2017, Prague, Czech Republic.

(c) 2017 Copyright held by the owner/author(s). 978-1-4503-4708-2/17/07 ..\$15.00 DOI: http://dx.doi.org/10.1145/3078714.3078718

\section{INTRODUCTION}

Hypertext has been described as a "computer-based medium for thinking and communication" [17]. It has a long tradition in computer science. Vannevar Bush foresaw the rapid growth of information and the need of associations already in the 1940s. He issued the idea that people should be able to persistently store "paths" among documents that can be followed at any time later or shared with others. He was pioneering the idea that human mind should be extended by machines and called his prototypic design Memory Extender or short Memex [15]. Later in the 1960s, when computers were more widely available in academia Ted Nelson coined "the word 'hypertext' to mean a body of written or pictorial material interconnected in such a complex way that it could not conveniently be presented or represented on paper" [41]. For the first time computers have been used to augment human intellect. In the following years Nelson's strategy was to develop a hypertext system that holds all documents and its versions and called it Xanadu [43]. Another hypertext pioneer was Douglas Engelbart, who developed Augment and NLS [25]. He had a cooperative point of view on hypertext as systems to be used for collaborative problem solving.

The 1980s became the time of various new hypertext projects, including KMS [1], Hyperties [56], NoteCards [30], Intermedia [37], Guide [14], and HyperCard [57]. They all followed a node-link paradigm and many of them were based on monolithic system architectures. The Dexter Hypertext Reference Model [28] aimed at providing a general model at the end of the pre-Web era.

In the late 1980s and early 1990s new structure types have been addressed by hypertext scientists, such as spatial hypertext [35], taxonomic hypertext [50], or argumentation supporting structures [18]. Those differed from the node-link structures fundamentally and demanded new system functionalities and behavior.

This was the birth of various research projects that aimed to provide generalized hypertext infrastructures, so-called Open Hypermedia Systems (OHS) and later Component-based OHS (CBOHS). Their aim is to abstract from specific structure services and enable specialized modules for the tasks at hand. Furthermore, they have the potential of interoperable services in order to support "functional aggregation of other domains" [59], for example, digital libraries and linguistic domains. 
This research on hypertext infrastructures has started fading out in the early 2000s, as Tim Berners-Lee's World Wide Web [10] became dominant in science and economy. Although there were approaches in merging OHS and the Web (i. e., [12]), the Web never adopted fundamental findings from hypertext research widely. A research gap can be witnessed between the original hypertext infrastructure work around the year 2000 and today which has not been compensated by the growing Web.

In this paper we describe our core system architecture as a consequent next step in the development of traditional hypertext infrastructures. Our major goal was to develop a component-based system that enables support for various structure types, including spatial hypertext, taxonomic hypertext, or node-link structures. From lessons learned we have significantly changed parts of traditional paradigms such that our system includes machine intelligence and provides better support to users.

The remainder of the paper includes a brief historical perspective of hypertext infrastructures in Sect. 2 which leads to a description of our current system in Sect. 3. In Sect. 4 we will present a case scenario taken from an ongoing research project. It acts as a proof of concept for the discussed infrastructure. Finally, Sect. 5 concludes our work and provides a glimpse of future work.

\section{HISTORICAL VIEW}

\subsection{General}

The evolution of hypertext systems can be described as successive steps of abstracting and opening up parts of their architecture $[46,48]$. Within each evolutionary step, such systems removed various functionalities from their core and made them identifiable, first class abstractions within their architectures. Being a first class abstraction, these functionalities could be reused and appropriately tailored to facilitate a variety of data structuring or organization problems. In the next paragraphs we outline the evolutionary steps hypermedia systems took from which their ability to address a wider range of structuring problems emerged.

\subsection{Monolithic Systems}

Early hypermedia systems are monolithic in their architecture (cf. Fig 1a). The term monolithic is used to denote that these systems do not identify or separate different layers and hence render presentation, hypertext, and storage layer as a single process. Those systems do not offer interfaces or protocols which the different layers could use to communicate. Most are based on node-link structures supporting navigation between informational units, for example, KMS [1], NoteCards [30], or Intermedia [69]. Some other monolithic systems support further structure domains, for example, argumentation support (e. g., gIBIS [19]) or spatial hypertext (e. g., VIKI [36], VKB [55], or Tinderbox [11]).

Due to their monolithic nature, these hypertext systems exhibited a number of shortcomings including:

(1) Proprietary document formats. In general, each monolithic hypertext system defines its own representation and storage format for hypertext documents. This requires the non-trivial task of transforming external data in order to be used [26].

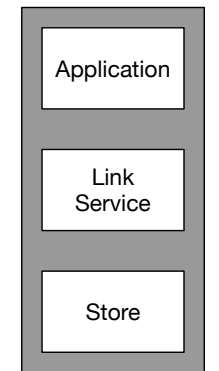

(a) Monolithic System

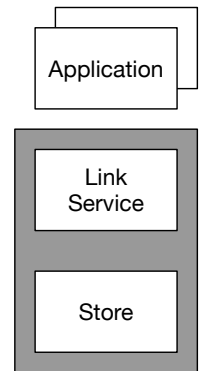

(b) Client-Server System

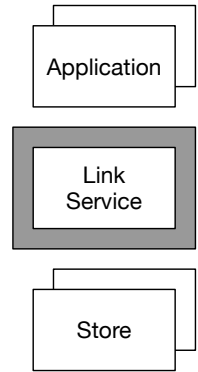

(c) Open Hypermedia System (OHS)

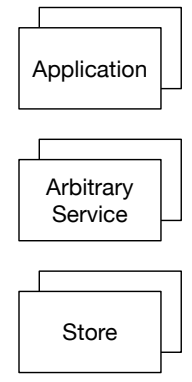

(d) Componentbased $\mathrm{OHS}$
Figure 1: Hypermedia infrastructure history [67]

(2) Embedded hypertext abstractions. Monolithic systems emphasize embedding hypertext abstractions (such as anchors, nodes, and links) within its content and storing them as a single entity. This makes it difficult for these systems to integrate other media.

(3) Closed set of applications. Their monolithic nature restrict development of new services and integration of third party applications. This forces users to abandon their preferred tools.

The above issues caused that monolithic hypertext systems are considered islands that would not "talk to each other" [60]. They are steering away from Ted Nelson's vision of a docuverse [42] and from making hypertext functionality as natural as "copy \& paste" in the everyday computing environment of users [38].

\subsection{Client Server Hypertext Systems}

The first move towards addressing the concerns regarding monolithic systems was to open up the application layer (cf. Fig 1b). This resulted in client-server based hypertext systems, in which an open set of applications could request hypertext functionalities from the system. In these systems applications (i. e., clients) request hypertext services from so-called link services which implement the hypertext layer by using specific system related protocols. Hence new client applications can be effortlessly developed by simply adhering to the system's protocol.

Client-server hypermedia systems can be classified as Link Server Systems (LSS) or Hyperbase Management Systems (HBMS). They differ in how they treat the storage of content. While for LSSs the data storage is handled by the application (not the hypertext system), in HBMSs it is the responsibility of the hypertext system [49]. HBMSs adopted such approach to address issues of editing problems or link integrity, whereby links still could point to data that has been deleted [20]. A notable example of a LSS is Sun's Link Service [51] while examples of HBMSs include Neptune [23], HAM [16], or DGS [54].

The World Wide Web [10] can also be considered a client-server hypertext system. However, while the WWW meets the mandatory requirement of offering an open set of applications that can communicate via a well defined protocol (namely HTTP), it has been criticized of its inability to "understand" and reason upon structural 
abstractions, such as anchors, nodes, and links. This makes the Web is very data (not structure) centric. Due to that the question has been raised of whether the WWW can be considered a hypertext system at all [45].

\subsection{Open Hypermedia Systems - OHS}

The next step for hypermedia systems was to further open up their architecture by decoupling the hypertext from the storage layer (cf. Fig 1c) as well as providing hypertext services to an even more extended set of applications, including third party applications. This development initiated the era of Open Hypermedia Systems (OHS). The focus of integrating third party applications was a focal point, attempting to bring hypermedia services to users' everyday computing environment [21,63].

These efforts gave birth to systems such as Devise [29] (demonstrating the integration of Microsoft Word with an hypermedia system), HyperDisco [66] (successfully integrating XEmacs), or Microcosm [32] (integrating Microsoft Calendar, WAIS, and Gopher). There were also efforts in integration the WWW with OHS (e. g., $[3,13])$.

OHSs ease the tailored integration of navigational hypertext functionality in a wide range of applications and avoid the need to design and implement large parts of the system over and over. Furthermore, standardization efforts were initiated in the context of the Open Hypermedia Systems Working Group (OHSWG) in order to support interoperability between applications and OHSs: applications integrated with one hypermedia system should be able to work with any other OHS as well. The Open Hypermedia Protocol (OHP), which specifies a standard way of communication between applications and hypermedia systems, was an outcome of these efforts [22].

The emphasis on integrating third party applications enabled OHSs to address problem areas that used to be outside the realm of hypertext. Examples include the fields of software engineering $[4,24]$ or ontologies [62] for which hypertext has been introduced as a useful way to organize and navigate these kind of information spaces. Despite their increasing openness regarding applications, OHSs still are systems that offer predominantly node-link structures to applications in order to facilitate navigation of information.

\subsection{Component-based Open Hypermedia Systems - CB-OHS}

Component-based Open Hypermedia Systems (CB-OHS) resulted from opening up the hypertext layer of OHSs (cf. Fig 1d). Several reasons led to this evolutionary step. One was that the design of the OHP turned out to be complicated [2]. Moreover, efforts were concentrated on supporting different structure paradigms within the same system, such as taxonomic or spatial hypertext as well as making their development and tailoring easier. The architecture of CB-OHS offers a hypertext layer that admits an open set of so-called structure services. Each of them addresses the organization problems of a particular domain, such as navigational, taxonomic, or spatial hypertext. Hence, a single CB-OHS is able to support an open set of organization problems in parallel. CB-OHSs have also reconsidered the storage layer, which is capable of storing generalized structures. This makes it possible to persistently store different hypertext types at the same place. This opened the discussion on how deep structure awareness should be pushed into the system, leading to the research thread of structural computing [44, 47].

There are different approaches of granting openness and generality for various CB-OHS layers; examples include Callimachus [59], the Fundamental Open Hypermedia Model (FOHM) [39, 40], or Construct [68]. The latter, for example, acknowledges any set of services at the hypertext layer, not only services related to hypertext functionality. This is why it has been classified as a Multiple Open Hypermedia System [65]. FOHM instead offers only support for three domains, namely navigational, spatial, and taxonomic. However, it devises for these distinct domains a well defined, coherent, and complete logical model for the hypertext layer.

As discussed above, hypermedia systems moved from closed, monolithic systems to open, distributed and modular systems, providing rich hypertext functionalities to an open set of applications. The benefits of CB-OHS include in particular (i) easier integration of new structure services; (ii) better bridging to Web-based services; (iii) efficient tailoring existing structure services for specific problem domains; and (iv) support of diverse data sources without the need for data transformation. These advantages will be demonstrated in the following sections.

\section{INFRASTRUCTURE DISCUSSION}

\subsection{General}

The design of previous hypertext systems was based upon the following specific priorities and assumptions:

(1) They were designed primarily with node-link structures in mind. Other structure types, such as spatial hypertext or metadata support (see, e. g., Construct [68]) have been included additionally on top of that.

(2) Sophisticated hypertext systems do not offer rich applications for various structure types. (See Wiil's keynote at ACM Hypertext 2005 [64]).

(3) Even though intelligence became a huge issue for the Web community, it has never been an important part of previous hypertext infrastructures.

However, today, we would expect hypertext systems that (i) provide sophisticated, component-based, and general purpose infrastructures; (ii) support various and rich structure types for different tasks at hand; and (iii) include intelligence components to support knowledge workers.

The research prototype described in this paper provides support for the following main features that are relevant for modern hypertext infrastructures:

(1) Component-based system with support for multiple structure domains (e. g., node-link structures, spatial hypertext, taxonomic hypertext, or argumentation support structures)

(2) General purpose infrastructure to be used in various application domains or for different organization problems

(3) Intelligence components for increased user support

(4) Multiple general purpose parsers to be used for spatial hypertext structures 
The overall framework is divided into three different areas with specific purposes. The names are borrowed from the Norse mythology:

Midgard includes all application components to which we mainly count user interfaces;

Asgard holds (partly intelligent) components that deal with structures, such as a spatial structure services with multiple, highly specialized parsers; and

Hel hosts a collection of knowledge-centric components, e. g., knowledge bases or intelligent software that is used to populate those.

The basic idea is that Asgard's components analyze structures that users created via Midgard applications. The result of this analysis is used to query relevant information from Hel knowledge bases which is then presented to the users.

The first developed Asgard component in our system was the spatial structure service. Instead of designing the overall architecture upon node-link structures (as it was the case with previous systems) the Asgard framework has been developed primarily with one of the most complex (and for machines most difficult to "understand") hypertext structures in mind. This approach appreciates the specific demands of implicit structures rather than explicit ones. As we will argue in the following, explicit structure types, such as node-link or taxonomic structures can be added easily. Those are currently under development.

Both a Hypertext System and a Visual Analytics Tool. Through Asgard and Hel the overall system reaches computational intelligence to a level not seen in previous traditional hypertext applications. This enables an iterative process of human and machine in which the machine learns from what the user does and the user benefits from the machine's suggestions. It can be seen as a way of intelligently navigating through knowledge provided by (i) the user's structure created in Midgard and computed in Asgard; and (ii) the machine computed knowledge existing in specialized Hel knowledge bases. As such, our system goes along with what is known as visual analytics and its mantra "Analyze first, Show the Important, Zoom, filter and analyze further, Details on demand." [34]. The relevance of Midgard, Asgard, and Hel for visual analytics point becomes even more obvious by a statement given by Sun et al.:

"The combination and interaction between visual and automatic analysis methods are the key feature of visual analytics, which helps distinguish the visual analytics process from other data analysis processes. It allows for progressive refinement and evaluation of the analysis results." [58]

We argue that from this perspective our framework can be considered both a generalized hypertext infrastructure and a specialized visual analytics tool.

Figure 2 provides an overview of potential infrastructure components. The communication protocols between system components are formally described in the Asgard technical specification [61] In the following sections we will present the three system layers individually, referring to the mentioned overview figure.

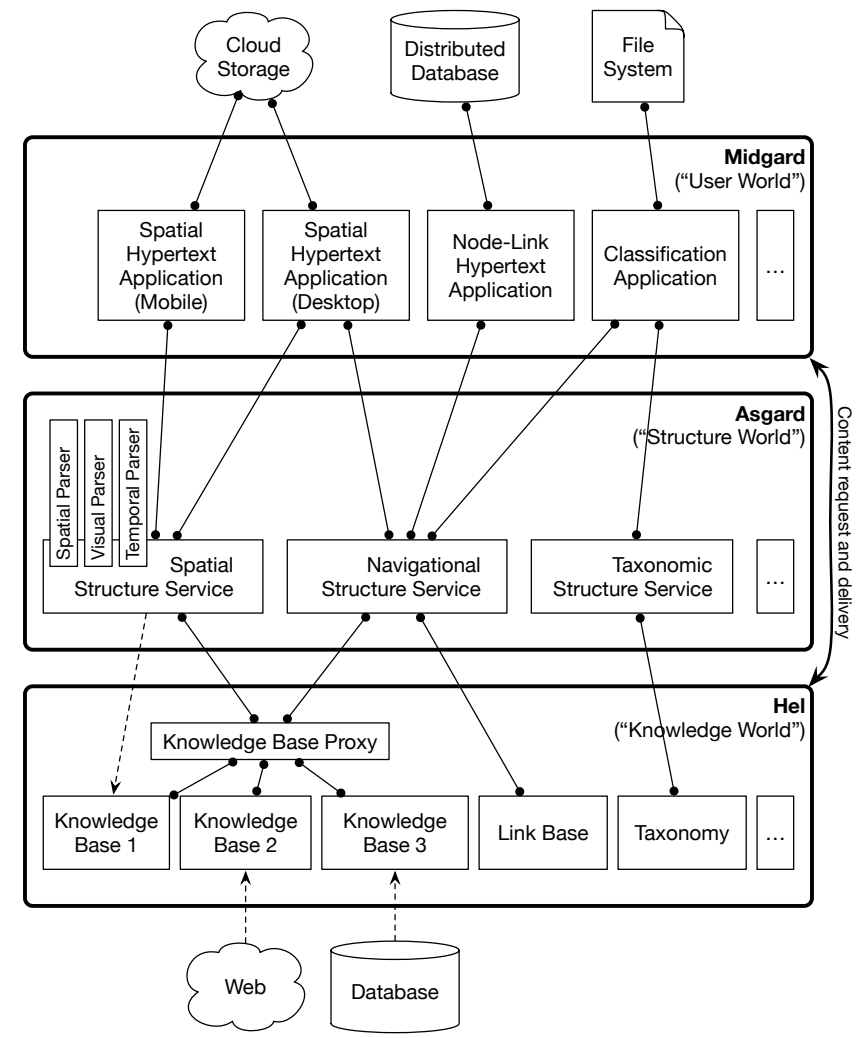

Figure 2: Infrastructure components in Midgard, Asgard, and Hel

\subsection{Asgard - The Structure World}

Asgard in Norse mythology is the place of the gods. In our system the name refers to the central intelligence components of the system. Those are components that deal with various kinds of structures and are aware of what is going on in the user's world. They also have access to knowledge-centric services. Asgard is the most central layer in our infrastructure. It refers to the arbitrary service layer in CB-OHSs [67] (cf. Fig. 1).

Today, Asgard provides a spatial structure service written in Java. Its structure awareness is provided by various parsers, including (i) a spatial parser that analyses the spatial arrangement or distance of objects; (ii) a visual parser that considers the visual appearance of objects; (iii) a temporal parser that computes relationships based on the sequence of user interactions; and (iv) a content parser that extracts relationships between objects based on their textual content. Those enable analyzing spatially organized information, as described in various previous work [cf. 7-9]. A novel approach was the consideration of time to foster higher accuracy in analyzed associations between informational units, which was first introduced in [6] and formally described in [52].

The spatial structure service follows a plug \& play metaphor for its parsers, such that others can be connected easily. As discussed in [53] the output of the various parsers is merged and normalized such that a high accuracy of correctly found relationships between spatially arranged objects is provided. 
The question remains about whether parsers (or similar components) should be treated as add-ons to structure services or rather be considered as top level Asgard components. We follow the paradigm to treat components as add-ons whenever they are specific to a single component, whereas software units that may be used by several components become native Asgard services. The mentioned parsers are highly specialized components, only used by the spatial structure service.

The spatial structure service is (as any Asgard service) purely structure aware, but does not have knowledge over the data itself. This idea is borrowed from the Dexter Hypertext Reference Model $[27,28,31]$ in which the so-called "storage layer" only considers structural components, but not the data within. The latter is taken care of inside the "within-component" layer. However, contrary to Dexter our infrastructure puts responsibility of managing data to Midgard applications. As a given spatial structure can be analyzed at any given time, there is no need to persistently store the computed associations (i. e., the parsers' output). Consequently, the spatial structure service has no facilities to save structure persistently. Instead, it holds the currently computed (i.e. yet made explicit) structure only in main memory.

Other Asgard services support node-link structures (so-called navigational structures) or taxonomies. Both (currently under development) differ in important aspects to spatial structure services. The most obvious fact is that they deal with explicit rather than implicit structures. As such, structure needs to be stored persistently in order to follow links later or classify objects according to a given taxonomy. In our architecture this is the task of specialized services within Hel (i. e., the "knowledge world") including link bases for navigational hypertext or storage facilities for taxonomies (see Fig. 2). From there the Asgard service retrieves information required for link traversal etc. This architecture consequently separates data (responsibility in Midgard) and structure/behavior (responsibility in Asgard/Hel).

Furthermore, there are connections between Hel knowledge bases and Asgard services. They are used to retrieve relevant information and pass it to the user interface. Indicated by a dashed arrow in Fig. 2, the spatial structure service is also able to fill a knowledge base with computed associations. This lets the overall system gain from user created knowledge.

\subsection{Midgard - The User World}

In Norse mythology Midgard is the place where humans live. In our system it refers to frontend applications, mainly to those used by humans to interact with the system. Those applications are also responsible themselves for persistently storing their data. This may be solved by writing it into files, connecting to additional databases, using cloud storage, etc.

It is assumed that any Midgard application supports structuring tasks, for example, via node-link hypertext, spatial hypertext, or classification tools. These applications are connected to the respective Asgard structure services. A Midgard application may connect to multiple structure services. For example, as indicated in Fig. 2, the spatial hypertext desktop application connects to both the spatial and navigational structure service. By doing so, the user may use spatial structuring and creating/traversing hypertext links within the same graphical user interface. This is what some monolithic spatial hypertext applications also can do (e. g., Tinderbox [11] or VKB [55]).

In particular spatial hypertext applications may demand a high frequency of messages being sent to or received from the spatial structure service. This is the case, for example, if during a drag operation of objects on the space the interim coordinates are communicated to Asgard. The effect that can be reached by that is astounding: while a user drags an object, suggested additional nodes coming from the Hel knowledge base fade in, smoothly reposition, or fade out. This enables the user to experiment with implicit associations in a very flexible way based on knowledge stored in Hel services. This feature is novel and unique in the context of hypertext systems. As discussed in Sect. 3.1, it shifts the overall system towards a specialized visual analytics tool that supports exploring unknown information spaces by an iterative process in which machine and human are involved equally.

In order to support the high frequency of messages sent between Midgard and Asgard, we developed our communication protocols to be in binary format. This is much more efficient than, for example, XML would be. Only the changes on a knowledge space are sent to Asgard. The suggested results coming from Hel are yet passed through Asgard to Midgard without further modification. However, those results do not contain content. This is natural for Asgard, as applications on this layer do not deal with content. From a Midgard perspective there are also good reasons for this:

(1) The content may be big (e. g., a picture), which would take some time to send to the GUI application. By receiving the associations of a suggested node beforehand, the GUI may calculate and indicate the positions of all suggested nodes to be placed on the screen before finishing loading their content.

(2) There may be too little space available to position all suggested nodes. For those discarded by the Midgard application no content request would be sent.

(3) During an operation (e. g., dragging a node) information from a knowledge base is added and immediately (i. e., even before the content is requested) removed again from space. In this case our approach shows higher efficiency.

Figure 2 indicates the direct connection between Midgard and Hel applications by an arrow on the right hand side.

The Midgard application is responsible for data storage, for example, a spatial hypertext, nodes of a navigational hypertext, or classified specimen. The storage itself takes place outside of our framework and depends on the application developer's decision. This could be storage facilities like (possibly distributed) file systems or databases, cloud storage, etc.

Mindspace and Mindspace2: Two Midgard Prototypes. Currently, two prototype Midgard application exist with the name of Mindspace (respectively Mindspace2), developed to demonstrate the basic concepts of the system from a user's perspective. The first prototype is written in Swift running on macOS, the latter (more sophisticated one) is written as a JavaFX application. Mindspace works as a virtual pin board allowing users to put nodes onto a $2 \mathrm{D}$ space. Furthermore, text can be added to nodes or visual attributes of nodes (e. g., size, shape, or color) can be changed. 


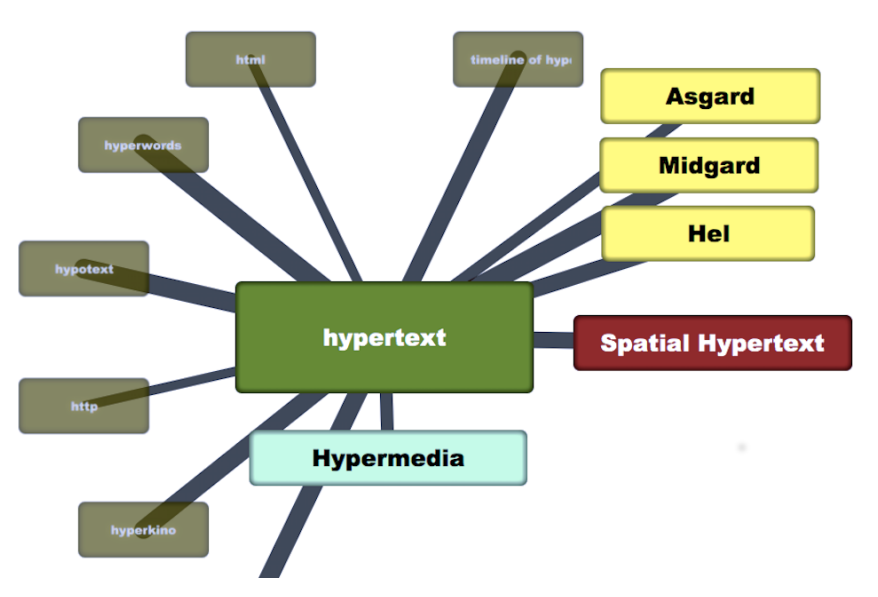

Figure 3: Hovering over a node (here: "hypertext") in Mindspace 2 fades in representations of associations to suggestion nodes including the connection strengths (i.e., line thickness)

Mindspace gathers these inputs and passes them to Asgard. The spatial structure service then parses those in order to identify associations between objects and query relevant information from $\mathrm{Hel}$. Asgard then sends back a set of suggestions as well as information about groupings that it has detected among the user's nodes.

The retrieved suggestion nodes are then displayed to the user. He may chose to turn some into "user nodes" by simply dragging them onto the space. Suggestion nodes may contain different data types. For example, a suggestion may contain a URI referencing a Web site, a JPEG image, etc. Currently, both Mindspace prototypes only support text or images, however, other data types may be included in the future.

Mindspace has a tight communication with $\mathrm{Hel}$ in order to exchanged data content as discussed above. Furthermore, it receives information about the strength between suggestion nodes and user added nodes from the knowledge base.

Hovering with the mouse over a node will show all currently known connections in Hel of this particular node to any other related node on the space. Figure 3 shows an example screenshot. The thickness of the respective lines correspond to the strength of the connection. With this information a user may get a quick overview on how current nodes are related.

In debug mode (cf. Fig. 4) additional information is displayed. This includes information about nodes' positions or their status in Midgard. In addition, this mode shows the group memberships computed by Asgard, indicated by colored borders surrounding each node. The same color indicates a membership of the same group.

Each suggestion node contains additional information that can be revealed by hovering over and clicking on the "text" icon. A result of that action can be seen in Fig. 5. Currently, only short Wikipedia excerpts are saved. However, also URIs to corresponding Wikipedia pages are available from within our system, which can be opened in a Web browser.

Finally, Mindspace currently stores its spatial hypertext persistently in files. On opening such a file, Mindspace replays the

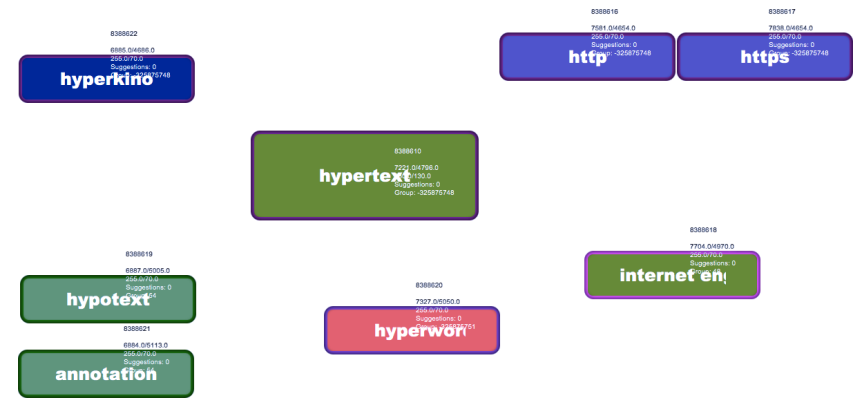

Figure 4: The debug mode (suggestions omitted) in Mindspace 2 shows additional information about nodes, including their group membership (computed by Asgard and indicated by colored borders around nodes)

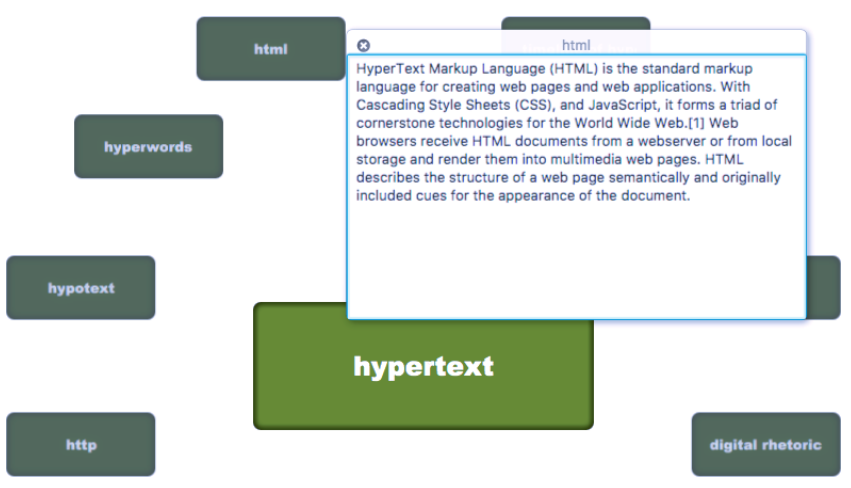

Figure 5: Viewing additional content associated with a suggestion node in Mindspace2 (in this example the related information comes from Wikipedia)

creation process and passes this information to Asgard. This enables Asgard to rebuild the interpretation of the structure, including analyzing the creation process itself (via the temporal parser). This is required, because Asgard does not persistently store computed structures (cf. Sect. 3.2).

\subsection{Hel - The Knowledge World}

The name Hel in Norse mythology refers to the death goddess and her realm alike. The name is related to the English word "hidden". In our terms it refers to knowledge-centric components that mostly include not yet explored information. It relies on the Asgard services to query relevant information and reveal it to the user via Midgard applications.

Previous traditional hypertext infrastructures do not deal with computer generated knowledge. This is a novel aspect of our system. Knowledge bases in Hel may be created upon various sources, depending on the target application/problem domain. This may include information from the Web (e. g., Wikipedia article or news sites), open data sites, databases with company related documents, or any other relevant source. Data and text mining methods are used to extract knowledge from these sources and store it in specific knowledge bases. Those knowledge bases may be of different type, including semantic databases or graph databases. The type depends 
on the requirements of the respective services, for example, the number of requests expected within a given time frame.

Knowledge bases may be also filled by knowledge extracted from user generated structures. Section 3.2 mentions spatial hypertexts as an example. Furthermore, link bases or taxonomies would be examples for Hel components, as they store (user generated) knowledge.

Figure 2 depicts also a knowledge base proxy. The reason behind is that the communication to general knowledge bases should become transparent to Asgard services. The proxy translates and distributes the queries to the available (i. e., registered) knowledge bases. The advantage is that a spatial structure service only has to implement a single interface in order to communicate with a heterogeneous set of knowledge bases, possibly each using a different query language.

Knowledge Base Implementations. Hel can be divided into three main parts: (i) the communication interface with Asgard; (ii) an interface towards Midgard; and (iii) the knowledge gathering components. Each of those components has different requirements towards the underlying structure of the databases. Changing the needs of the overall system may require modified protocols or even different databases.

Even though pure relational databases show a very good sequential search performance, they cause problems in our system. An object within the database should be able to have any number of properties, ideally without type constraints. Thus NoSQL documentbased databases seem to be a good choice, as they provide a good performance and support arbitrary properties. However, representing connections between objects can be done more efficiently in graph databases.

Currently, Hel includes two implemented databases: (i) the graph database $N e 04 j^{1}$; and (ii) the NoSQL document database MongoDB ${ }^{2}$. The reason behind picking those two databases for implementation and demonstration was to get the best of the two worlds: while MongoDB would be used to store documents corresponding to any given object, Neo4j would hold the corresponding graph (i. e., connections between those objects). This increased the overall performance for graph-based requests. Furthermore, MongoDB overcame performance issues we had encountered by exclusively emulating documents on Neo4j's property system.

While in current protocols the functionality requirements towards Hel are intentionally very limited, more complex requests can be easily adapted. To support this, Hel offers a proxy layer described above. In its current version the proxy distributes incoming requests to all connected databases, merges all incoming replies into a single message, and returns it to Asgard.

In order to fill the knowledge bases with useful information we first targeted Wikipedia and used basic data mining methods, for example, the Seealsology tool ${ }^{3}$, which uses ForceAtlas2 [33] for generating a graph of Wikipedia. From this graph (based on the distances between any two nodes), weights have been computed. Then, the full weighted graph has been added to the Neo4j database.

\footnotetext{
$\overline{{ }^{1} \text { https://neo4j.com/ }}$

${ }^{2}$ https://www.mongodb.com/

${ }^{3} \mathrm{http}: / /$ tools.medialab.sciences-po.fr/seealsology/
}

Additionally, the MongoDB database has been filled with the corresponding documents, each one representing a single Wikipedia page.

\section{THE ODIN/HEIMDALL PROJECT}

\subsection{About the Project}

In this section we describe a scenario that reflects the use of our framework in the domain of software engineering (partly still under development). It is also a proof of concept that shows that our framework is capable of working with other (in this case Webbased) services.

There are various possibilities for publishing data on the World Wide Web, but in most cases they are difficult to use. To simplify this process is the main goal of the ODIN ("Open Data Innovation") project. Its sub-project HEIMDALL focuses on an intelligent user interface. ODIN uses a Web-based platform. Instead of harvesting data from many different data sources (with all accompanying problems like different file formats or access possibilities) ODIN offers ready-to-use components for accessing, manipulating, and visualizing data. Those are called Cubbles; they are based on Meme Media Technology and previously known as Webbles [cf. 5]. Users can utilize those or publish new ones by uploading them to a public server, the so-called Cubbles Base. Cubbles are Web components, implemented in JavaScript and executed in the user's Web browser (which loads all required Cubbles from the Cubbles Base.) This makes Cubbles highly reusable software components.

The composition of already existing Cubbles components can be done in a classic manner, for example, by using an IDE. An alternative approach would be the use of the $\mathrm{BDE}^{4}$ ("Browser-based Development Environment"), a Web application offering a 2D space on which Cubbles (represented as rectangles with names) can be added and connected. This eases data processing tasks in particular for non-programmers.

For example, imagine a scenario in which a researcher wants to correlate weather data and data about traffic accidents in order to publish the result on a Web page. Even though he finds appropriate data sources, he may not be able to transform the data and generate a meaningful visualization as he is not an IT expert. Assuming that the Cubbles Base already contains all required components, he would just have to add them to the work space and connect them to model the desired data flow.

\subsection{Cubbles Integration in the Hypertext Infrastructure}

The current implemented solution of the Cubbles Web application lacks an efficient way of finding required components. We want to support the user by proposing other relevant Cubbles. For that an Asgard service is used to analyze the user created spatial structure and matching Cubbles will then be presented to the user. Those can then be easily added and used. Figure 6 shows the BDE including some Cubbles that model the above mentioned scenario. The system suggests some further components suitable for the task at hand.

In addition to the solution of the described problem, we wanted to demonstrate how our infrastructure can easily be used for various

$\overline{{ }^{4} \text { https://github.com/cubbles/bde }}$ 


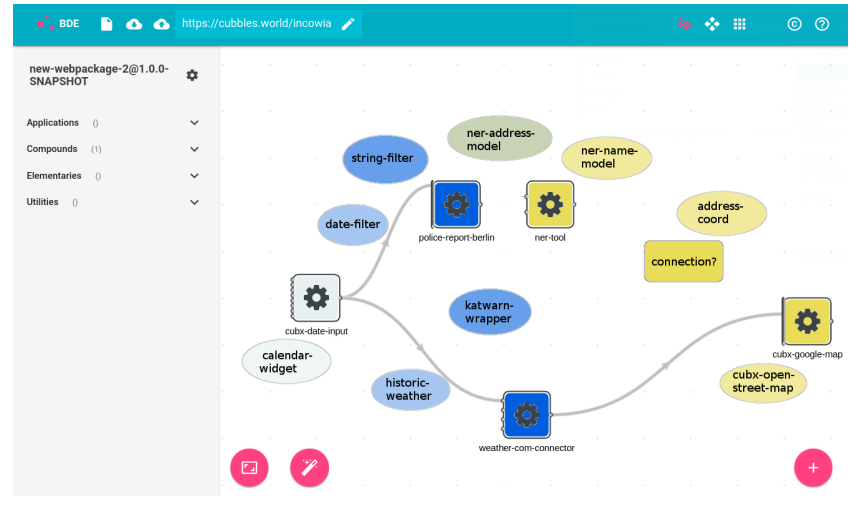

Figure 6: BDE work space with Cubbles and suggestions

scenarios. Since we use a three layer software architecture, the BDE can be seen as a Midgard implementation. Because Asgard and Hel host multiple services, there is no need to re-implement any business logic in the Midgard layer. Furthermore, there is no need for customizing, since we use generic messaging between layers [cf. 61].

The loosely coupled layers make it possible to be independent of specific programming languages, as long as they support TCP/IP communication. However, even though JavaScript is not limited to that respect, Web browsers are. We needed a solution that would work with the protocols defined in [61]. One possibility would be a Web service using simple HTTP requests, offering a second API to Asgard and Hel. This approach would ease the development of a client written in JavaScript or another language, however, using our low level protocols would become difficult. As we wanted to strictly stick to the given architecture and its layers as well as to the TCP/IP based protocols, we decided to follow a different path.

The messages between components via TCP/IP reduce messaging overhead and feature a full duplex communication between client and server. To make use of these advantages we chose to establish a connection via WebSocket ${ }^{5}$, a protocol supported by the majority of modern Web browsers, set up on top of TCP. With WebSocket it is not necessary to define a new API connecting Midgard to the other layers; a script called "websockify"6 translates WebSocket traffic to normal socket traffic. This shows the flexibility of low level protocols. The required JavaScript code is designed in a way to work also with any future projects. It hides the protocol behind user-friendly and generic method calls.

Besides the technical integration, it is important that Asgard and $\mathrm{Hel}$ are capable of handling the specific needs issued in the ODIN project. Sect. 3.2 mentions that Asgard is only aware of structure, but not of data. Thus, it does not matter whether text nodes, pictures, or Cubbles are used on the space. A big challenge, however, is building a suitable knowledge base for the Cubbles Base which holds associations between Cubbles. Those are then used to identify the relevant ones.

Cubbles are rather complex in their characteristics. They have many different properties to be considered for appropriate feature

\footnotetext{
${ }_{5 \text { https://tools.ietf.org/html/rfc6455 }}$

${ }^{6}$ https://github.com/novnc/websockify
}

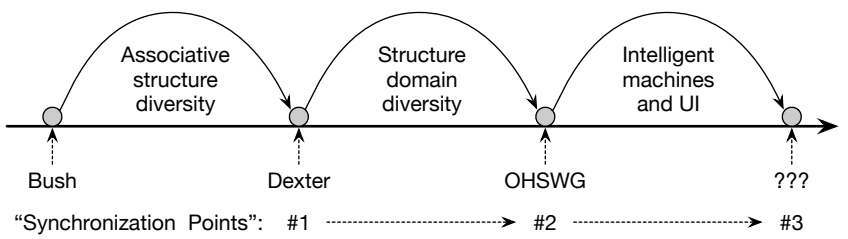

Figure 7: Synchronization points in hypertext research, extending [64]

vectors. This work has to be done for every scenario, since there is no "omniscient" knowledge base. The advantage of our architecture is the independence of Asgard regarding these project specific issues. This allows us to focus on functional integration, making use of the already existing spatial structure service. Furthermore, Asgard/Hel does not restrict the design and technical implementation of the knowledge base; as discussed in Sect. 3.4, we can pick a database that fits the requirements of the project best.

\section{CONCLUSION AND FUTURE WORK}

In his 2005 ACM Hypertext conference keynote Wiil addressed:

"In order to reach the full potential of hypermedia technology support for knowledge workers, a long term community effort $[. .$.$] is needed - an effort$ where domain experts and infrastructure experts work together to fulfill a common vision." [64]

He refers to certain "synchronization points" in hypertext research, depicted in Fig. 7. The first took place when the Dexter Hypertext Reference Model was defined, the second when the OHSWG defined protocols for open hypermedia (cf. Sect. 1 and 2). Exactly one decade after that keynote we now extend the original figure by adding machine intelligence and intelligent user interfaces to it. This defines the next (not yet named) synchronization point \#3.

We argue that the infrastructure proposed in this paper is a demonstration expected for synchronization point \#3, as we combine "fancy infrastructure" and "fancy applications", as suggested in [64]. Additionally, we push intelligence into the system, which is currently discussed in various fields, such as big/smart data, visual analytics, or artificial intelligence. In particular we argue that our system

(1) provides a flexible and modular infrastructure supporting various services and structure domains;

(2) is designed from the beginning with spatial hypertext in mind, the most difficult hypertext structure type to be handled by machines;

(3) includes sophisticated parsers for spatial hypertext structures that form fundamental modules for rich user interfaces; and

(4) makes heavy use of knowledge bases built by supporting intelligence components.

There are a number of issues still to be solved by future work. Those can be divided in three different categories: (i) improving or extending the infrastructure; (ii) identifying application domains 
and creating matching user interfaces; and (iii) designing and building up specialized knowledge bases that match the demands of the target application domains.

In more detail, the development of additional structure services (e. g., node-link or taxonomy support) needs to be finished. Also, the ODIN/HEIMDALL software needs to be completed with respect to their full feature sets. New parsers should be introduced, for example, the content parser (computing associations based on nodes' content). This opens again the question of how to prioritize various parser outputs that are running in parallel. Adaptive features may be considered to better focus on the users' needs. Research provided by the adaptive hypertext community will be of high value to this. Furthermore, we plan to provide clients for mobile devices (e. g., mobile phones or tablets). This opens the discussion of multi-structure support on multitouch devices. A still not solved question relates to security and privacy issues, which would need to be solved urgently if the system is used in productive environments.

As argued, there are a number of application domains that may benefit from the presented infrastructure. In the ODIN/HEIMDALL project we focus already on software engineering tasks. Furthermore, we consider e-learning, since linking of information as well as emerging structures (nicely to build up with spatial hypertexts) are important aspects for learners.

We are currently looking into using our system in combination with specialized user interfaces for people with dementia. First discussions with experts supports us in our assumptions that rich and intelligent structures may support those patients in their daily tasks and provide a means to physicians for diagnosing or measuring the progress of the disease.

Another area we are currently looking into is the field of "Industry 4.0 ". We plan to build maintenance diagnosis software on top of our infrastructure that provides linking and implicit associations (via spatial hypertext). Experts will be enabled to express their tacit knowledge at ease. We plan to enrich this with machine intelligence to further support users.

Finally, we need to spend time for identifying the best database technology for the specific knowledge bases in the context of their target application domains and tasks at hand. Furthermore, we have to identify good candidates for data sources and content processing in order to fill these knowledge bases, which depends on the respective application domain. Another important question relates to the computed associations within the knowledge bases, which directly influences the feedback proposed to the users.

As expected from a system that is as complex and rich as the proposed one, there a many open questions. It is up the scientific community to address those within the next years. There is a high potential of such systems to be used in many different contexts for the benefit of individuals and society.

\section{ACKNOWLEDGMENTS}

This work is part of the ODIN project, sub-project HEIMDALL, funded by the German Federal Ministry of Education and Research (grant ID 03PSWKPD).

\section{REFERENCES}

[1] Robert M. Akscyn, Donald L. McCracken, and Elise A. Yoder. 1988. KMS: a distributed hypermedia system for managing knowledge in organizations. Commun.
ACM 31, 7 (1988), 820-835. http://doi.acm.org/10.1145/48511.48513

[2] Kenneth Anderson, Richard Taylor, and E. Whitehead. 2006. A Critique of the Open Hypermedia Protocol. Fournal of Digital Information 1, 2 (2006). https: //journals.tdl.org/jodi/index.php/jodi/article/view/5

[3] Kenneth M. Anderson. 1997. Integrating Open Hypermedia Systems with the World Wide Web. In Proceedings of the Eighth ACM Conference on Hypertext. ACM, 157-166. http://doi.acm.org/10.1145/267437.267454

[4] Kenneth M. Anderson. 1999. Supporting software engineering with open hypermedia. ACM Computing Surveys (CSUR) 31, 4es (1999), 20. http://doi.acm.org/10. $1145 / 345966.346013$

[5] Oksana Arnold, Wolfgang Spickermann, Nicolas Spyratos, and Yuzuru Tanaka (Eds.). 2013. Webble Technology. First Webble World Summit (WWS). Communications in Computer and Information Science, Vol. 372. Springer.

[6] Claus Atzenbeck and David L. Hicks. 2009. Integrating Time Into Spatially Represented Knowledge Structures. In Proceedings of the International Conference on Information, Process, and Knowledge Management (eKNOW). IEEE Computer Society, 34-42. http://dx.doi.org/10.1109/eKNOW.2009.27

[7] Claus Atzenbeck, David L. Hicks, and Nasrullah Memon. 2008. Emergent Structure and Awareness Support for Intelligence Analysis. In Proceedings of the 12th International Conference on Information Visualization (IV'08), Ebad Banissi, Liz Stuart, Mikael Jern, Gennady Andrienko, Francis T. Marchese, Nasrullah Memon, Reda Alhajj, Theodor G. Wyeld, Remo Aslak Burkhard, Georges Grinstein, Dennis Groth, Anna Ursyn, Carsten Maple, Anthony Faiola, and Brock Craft (Eds.). IEEE Computer Society, 326-332. http://dx.doi.org/10.1109/IV.2008.44

[8] Claus Atzenbeck, David L. Hicks, and Nasrullah Memon. 2011. Supporting Reasoning and Communication for Intelligence Officers. International fournal of Networking and Virtual Organisations (IfNVO), Special Issue on Open Source Intelligence and Web Mining (OSINT-WM) 8, 1/2 (2011). http://dx.doi.org/10.1504/ IJNVO.2011.037159

[9] Claus Atzenbeck, Fatih Ozgul, and David L. Hicks. 2009. Linking and Organising Information in Law Enforcement Investigations. In Proceedings of the 13th International Conference on Information Visualization (IV'09). IEEE Computer Society, 443-449. http://dx.doi.org/10.1109/IV.2009.107

[10] Tim Berners-Lee, Robert Cailliau, Ari Luotonen, Henrik Frystyk Nielsen, and Arthur Secret. 1994. The World-Wide Web. Communication of the ACM 37, 8 (Aug. 1994), 76-82. http://doi.acm.org/10.1145/179606.179671

[11] Mark Bernstein. 2006. The Tinderbox Way. Eastgate Systems.

[12] Niels Olof Bouvin. 2000. Augmenting the Web through Open Hypermedia. The Development of the Arakne Environment, a Collaborative Open Hypermedia System for Web Augmentation. Ph.D. Dissertation. University of Aarhus. http://www. daimi.au.dk/ bouvin/Arakne/thesis.pdf

[13] Niels Olof Bouvin. 2000. Experiences with OHP and Issues for the Future. In Proceedings of the 6th International Workshop and 2nd International Workshop on Open Hypertext Systems and Structural Computing. Springer, 13-22. http: //www.springerlink.com/link.asp?id=du610p0bt7xnqrca

[14] P. J. Brown. 1987. Turning ideas into products: the Guide system. In Proceedings of the ACM Conference on Hypertext. ACM, 33-40. http://doi.acm.org/10.1145/ 317426.317430

[15] Vannevar Bush. 1945. As we may think. The Atlantic Monthly 176, 1 (7 1945), 101-108. http://www.theatlantic.com/doc/194507/bush

[16] Brad Campbell and Joseph M. Goodman. 1988. HAM: a general purpose hypertext abstract machine. Commun. ACM 31, 7 (1988), 856-861. http://doi.acm.org/10. $1145 / 48511.48515$

[17] Jeff Conklin. 1987. Hypertext: an introduction and survey. Computer 20, 9 (1987), 17-41. http://dx.doi.org/10.1109/MC.1987.1663693

[18] Jeff Conklin and Michael L. Begeman. 1987. gIBIS: a hypertext tool for team design deliberation. In Proceedings of the ACM Conference on Hypertext. ACM Press, 247-251. http://doi.acm.org/10.1145/317426.317444

[19] Jeff Conklin and Michael L. Begeman. 1988. gIBIS: a hypertext tool for exploratory policy discussion. In Proceedings of the 1988 ACM Conference on ComputerSupported Cooperative Work. ACM Press, 140-152. http://doi.acm.org/10.1145/ 62266.62278

[20] Hugh Davis, Wendy Hall, Ian Heath, Gary Hill, and Rob Wilkins. 1992. Towards an Integrated Information Environment with Open Hypermedia Systems. In Proceedings of the ACM Conference on Hypertext. ACM, 181-190. http://doi.acm. org/10.1145/168466.168522

[21] Hugh C. Davis, Simon Knight, and Wendy Hall. 1994. Light Hypermedia Link Services: A Study of Third Party Application Integration. In Proceedings of the 1994 ACM European Conference on Hypermedia Technology. ACM, 41-50. http://doi.acm.org/10.1145/192757.192767

[22] H. C. Davis, D. E. Millard, S. Reich, N. Bouvin, K. Grønbæk, P. J. Nürnberg, L. Sloth, U. K. Wiil, and K. Anderson. 1999. Interoperability between hypermedia systems: the standardisation work of the OHSWG. In Proceedings of the 10th ACM Conference on Hypertext and Hypermedia. ACM Press, 201-202. http://doi. acm.org/10.1145/294469.294904

[23] Norman Delisle and Mayer Schwartz. 1986. Neptune: a hypertext system for CAD applications. In Proceedings of the 1986 ACM SIGMOD international conference on Management of data. ACM Press, 132-143. http://doi.acm.org/10.1145/16894. 
16867

[24] Serge Demeyer. 1996. ZYPHER - Tailorability as a Link from Object-Oriented Software Engineering to Open Hypermedia. Ph.D. Dissertation. Vrije University.

[25] Douglas C. Engelbart. 1962. Augmenting Human Intellect: A Conceptual Framework. Summary Report AFOSR-3233. Standford Research Institute. http: //www.dougengelbart.org/pubs/augment-3906.html

[26] A. Fountain, W. Hall, I. Heath, and H. Davis. 1990. Microcosm: An Open Model for Hypermedia with Dynamic Linking. In Hypertext: Concepts, Systems and Applications, Proceedings of ECHT'90, A. Rizk, N. Streitz, and J. Andre (Eds.). Cambridge University Press, 298-311. http://eprints.soton.ac.uk/254308/

[27] Kaj Grønbæk, Jens A. Hem, Ole L. Madsen, and Lennert Sloth. 1994. Cooperative hypermedia systems: a Dexter-based architecture. Commun. ACM 37, 2 (1994), 64-74. http://doi.acm.org/10.1145/175235.175240

[28] Kaj Grønbæk and Randall H. Trigg. 1992. Design issues for a Dexter-based hypermedia system. In Proceedings of the ACM Conference on Hypertext. ACM Press, 191-200. http://doi.acm.org/10.1145/168466.168525

[29] Kaj Grønbæk and Randall H. Trigg. 1994. Design issues for a Dexter-based hypermedia system. Commun. ACM 37, 2 (2 1994), 40-49. http://doi.acm.org/10. $1145 / 175235.175238$

[30] Frank G. Halasz, Thomas P. Moran, and Randall H. Trigg. 1987. NoteCards in a nutshell. In Proceedings of the SIGCHI/GI Conference on Human Factors in Computing Systems and Graphics Interface (CHI '87). ACM Press, 45-52. http: //doi.acm.org/10.1145/29933.30859

[31] Frank G. Halasz and Mayer Schwartz. 1994. The Dexter Hypertext Reference Model. Commun. ACM 37, 2 (2 1994), 30-39. http://doi.acm.org/10.1145/175235. 175237

[32] Gary Hill and Wendy Hall. 1994. Extending the microcosm model to a distributed environment. In Proceedings of the 1994 ACM European Conference on Hypermedia Technology. ACM Press, 32-40. http://doi.acm.org/10.1145/192757.192763

[33] Mathieu Jacomy, Tommaso Venturini, Sebastien Heymann, and Mathieu Bastian. 2014. ForceAtlas2, a Continuous Graph Layout Algorithm for Handy Network Visualization Designed for the Gephi Software. PLOS ONE 9, 6 (June 2014), 1-12. http://dx.doi.org/10.1371/journal.pone.0098679

[34] Daniel Keim, Gennady Andrienko, Jean-Daniel Fekete, Carsten Görg, Jörn Kohlhammer, and Guy Melançon. 2008. Visual Analytics: Definition, Process, and Challenges. In Information Visualization, Andreas Kerren, JohnT. Stasko, Jean-Daniel Fekete, and Chris North (Eds.). Lecture Notes in Computer Science, Vol. 4950. Springer Berlin Heidelberg, 154-175. http://dx.doi.org/10.1007/ 978-3-540-70956-5_7

[35] Catherine C. Marshall, Frank G. Halasz, Russell A. Rogers, and William C. Janssen. 1991. Aquanet: a hypertext tool to hold your knowledge in place. In Proceedings of the 3rd ACM Conference on Hypertext. ACM Press, 261-275. http://doi.acm. org/10.1145/122974.123000

[36] Catherine C. Marshall, Frank M. Shipman, and James H. Coombs. 1994. VIKI Spatial hypertext supporting emergent structure. In Proceedings of the 1994 ACM European Conference on Hypermedia Technology. ACM Press, 13-23. http: //doi.acm.org/10.1145/192757.192759

[37] Norman Meyrowitz. 1986. Intermedia: The architecture and construction of an object-oriented hypemedia system and applications framework. ACM SIGPLAN Notices 21, 11 (1986), 186-201. http://doi.acm.org/10.1145/960112.28716

[38] Norman Meyrowitz. 1989. The missing link: why we're all doing hypertext wrong. In The society of text: hypertext, hypermedia, and the social construction of information. MIT Press, 107-114.

[39] David E. Millard. 2003. Discussions at the data border: from generalised hypertext to structural computing. Fournal of Network and Computer Applications 26, 1 (2003), 95-114. http://dx.doi.org/10.1016/S1084-8045(02)00063-2

[40] Dave E. Millard, Luc Moreau, Hugh C. Davis, and Siegfried Reich. 2000. FOHM: A Fundamental Open Hypertext Model for Investigating Interoperability between Hypertext Domains. In Proceedings of the 11th ACM Conference on Hypertext and Hypermedia. ACM Press, 93-102. http://doi.acm.org/10.1145/336296.336334

[41] T. H. Nelson. 1965. Complex information processing: a file structure for the complex, the changing and the indeterminate. In Proceedings of the 20th National Conference. ACM Press, 84-100. http://doi.acm.org/800197.806036

[42] Theodor Holm Nelson. 1982. Literary Machines. Mindful Press.

[43] Theodor Holm Nelson. 1999. The Unfinished Revolution and Xanadu. Comput. Surveys 31, 4es (Dec. 1999). http://doi.acm.org/10.1145/345966.346039

[44] Peter J. Nürnberg. 1997. HOSS: An Environment to Support Structural Computing. Ph.D. Dissertation. Department of Computer Science, Texas A\&M University. http://cs.aue.auc.dk/ pnuern/papers/diss/

[45] Peter J. Nürnberg and Helen Ashman. 1999. What was the question? Reconciling open hypermedia and World Wide Web research. In Proceedings of the 10th ACM Conference on Hypertext and Hypermedia. ACM Press, 83-90. http://doi.acm.org/ $10.1145 / 294469.294492$

[46] Peter J. Nürnberg, John J. Leggett, and Erich R. Schneider. 1997. As we should have thought. In Proceedings of the 8th ACM Conference on Hypertext. ACM Press, 96-101. http://doi.acm.org/10.1145/267437.267448

[47] Peter J. Nürnberg, John J. Leggett, Erich R. Schneider, and John L. Schnase. 1996 Hypermedia operating systems: a new paradigm for computing. In Proceedings of the 7th ACM Conference on Hypertext. ACM Press, 194-202. http://doi.acm. org $/ 10.1145 / 234828.234847$

[48] Peter J. Nürnberg, John J. Leggett, and Uffe K. Wiil. 1998. An agenda for open hypermedia research. In Proceedings of the 9th ACM Conference on Hypertext and Hypermedia. ACM Press, 198-206. http://doi.acm.org/10.1145/276627.276649

[49] Kasper Østerbye and Uffe Kock Wiil. 1996. The flag taxonomy of open hypermedia systems. In Proceedings of the 7th ACM Conference on Hypertext. ACM Press, 129139. http://doi.acm.org/10.1145/234828.234841

[50] H. Van Dyke Parunak. 1991. Don't link me in: Set based hypermedia for taxonomic reasoning. In Proceedings of the 3rd Annual ACM Conference on Hypertext. ACM Press, 233-242. http://doi.acm.org/10.1145/122974.122998

[51] Amy Pearl. 1989. Sun's Link Service: a protocol for open linking. In Proceedings of the 2nd Annual ACM Conference on Hypertext. ACM Press, 137-146. http: //doi.acm.org/10.1145/74224.74236

[52] Thomas Schedel. 2016. Spatio-Temporal Parsing in Spatial Hypermedia. Ph.D. Dissertation. Aalborg University. https://opus4.kobv.de/opus4-hof/frontdoor/ index/index/docId/71

[53] Thomas Schedel and Claus Atzenbeck. 2016. Spatio-Temporal Parsing in Spatial Hypermedia. In Proceedings of the 27th ACM Conference on Hypertext and Social Media. ACM, 149-157. http://dx.doi.org/10.1145/2914586.2914596

[54] Douglas E. Shackelford, John B. Smith, and F. Donelson Smith. 1993. The Architecture and Implementation of a Distributed Hypermedia Storage System. In Proceedings of the Fifth ACM Conference on Hypertext. New York, NY, USA, 1-13. http://doi.acm.org/10.1145/168750.168753

[55] Frank M. Shipman, Robert Airhart, Haowei Hsieh, Preetam Maloor, J. Michael Moore, and Divya Shah. 2001. Visual and spatial communication and task organization using the Visual Knowledge Builder. In Proceedings of the 2001 International ACM SIGGROUP Conference on Supporting Group Work. ACM Press, 260-269. http://doi.acm.org/10.1145/500286.500325

[56] Ben Shneiderman. 1987. User interface design for the Hyperties electronic encyclopedia (panel session). In Proceedings of the ACM conference on Hypertext. ACM Press, 189-194. http://doi.acm.org/10.1145/317426.317441

[57] Ted Smith and Steve Bernhardt. 1988. Expectations and experiences with HyperCard: a pilot study. In Proceedings of the 6th Annual International Conference on Systems Documentation (SIGDOC '88). ACM Press, 47-56. http://doi.acm.org/10. $1145 / 358922.358931$

[58] Guo-Dao Sun, Ying-Cai Wu, Rong-Hua Liang, and Shi-Xia Liu. 2013. A Survey of Visual Analytics Techniques and Applications: State-of-the-Art Research and Future Challenges. Journal of Computer Science and Technology 28, 5 (9 2013), 852-867. http://link.springer.com/article/10.1007/s11390-013-1383-8

[59] Manolis Tzagarakis, Dimitris Avramidis, Maria Kyriakopoulou, Monica M. C. Schraefel, Michalis Vaitis, and Dimitris Christodoulakis. 2003. Structuring primitives in the Callimachus component-based open hypermedia system. Fournal of Network and Computer Applications 26, 1 (2003), 139-162. http://dx.doi.org/10. 1016/S1084-8045(02)00064-4

[60] Andries van Dam. 1988. Hypertext '87: Keynote Address. Commun. ACM 31, 7 (July 1988), 887-895. http://doi.acm.org/10.1145/48511.48519

[61] Visual Analytics Research Group. 2017. Asgard Core. Technical Specification rev. f2d1ab62a824. Institute of Information Systems at Hof University.

[62] Mark J. Weal, Gareth V. Hughes, David E. Millard, and Luc Moreau. 2001. Open hypermedia as a navigational interface to ontological information spaces. In Proceedings of the 12th ACM Conference on Hypertext and Hypermedia. ACM Press, 227-236. http://doi.acm.org/10.1145/504216.504270

[63] E. James Whitehead. 1997. An Architectural Model for Application Integration in Open Hypermedia Environments. In Proceedings of the Eighth ACM Conference on Hypertext. ACM, 1-12. http://doi.acm.org/10.1145/267437.267438

[64] Uffe Kock Wiil. 2005. Hypermedia technology for knowledge workers: a vision of the future. In Proceedings of the 16th ACM Conference on Hypertext and Hypermedia. ACM Press, 4-6. http://doi.acm.org/10.1145/1083356.1083358

[65] Uffe K. Wiil, David L. Hicks, and Peter J. Nürnberg. 2001. Multiple open services: a New Approach to Service Provision in Open Hypermedia Systems. In Proceedings of the 12th ACM Conference on Hypertext and Hypermedia. ACM Press, 83-92. http://doi.acm.org/10.1145/504216.504241

[66] Uffe Kock Wiil and John J. Leggett. 1996. The HyperDisco approach to open hypermedia systems. In Proceedings of the 7th ACM Conference on Hypertext. ACM Press, 140-148. http://doi.acm.org/10.1145/234828.234842

[67] Uffe K. Wiil, Peter J. Nürnberg, and John J. Leggett. 1999. Hypermedia research directions. An infrastructure perspective. Comput. Surveys 31, 4 (12 1999). http: //doi.acm.org/10.1145/345966.345971

[68] Uffe K. Wiil, Samir Tata, and David L. Hicks. 2003. Cooperation services in the Construct structural computing environment. Journal of Network and Computer Applications 26, 1 (2003), 115-137. http://dx.doi.org/10.1016/S1084-8045(02) 00065-6

[69] Nichole Yankelovich, Bernard J. Haan, Norman K. Meyrowitz, and Steven M. Drucker. 1988. Intermedia: The Concept and the Construction of a Seamless Information Environment. Computer 21, 1 (1988), 81-83, 90-96. http://dx.doi. $\operatorname{org} / 10.1109 / 2.222120$ 